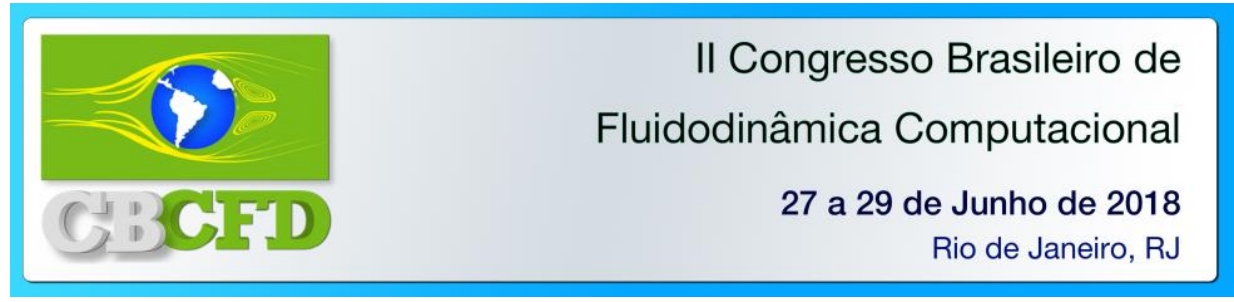

\title{
SIMULAÇÃO DE UM BIOFILTRO DE LEITO FLUIDIZADO PARA A AQUICULTURA
}

\author{
S. E. R. LIMA ${ }^{1}$, E. R. ALMEIDA ${ }^{1}$, E. P. FERREIRA ${ }^{1}$, T. F. PEREIRA ${ }^{1}$, R.S.T. de MOURA ${ }^{1}$ \\ e M. C. S. SANT'ANNA ${ }^{1}$
${ }^{1}$ Centro de Ciências Humanas, Naturais, Saúde e Tecnologia da Universidade Federal do Maranhão - Campus Pinheiro, Curso de Engenharia de Pesca \\ E-mail para contato: sandy.evelin.se@gmail.com
}

A aquicultura tem aumentado intensamente nos últimos anos e para alcançar o desenvolvimento que se espera, muitos desafios precisam ser superados, a exemplo da utilização da água, alvo de preocupação mundial. Os biofiltros de areia fluidizada possuem tecnologia eficiente e competitiva em termos de custos para a remoção de resíduos dissolvidos nesses tipos de sistemas. O objetivo deste trabalho é utilização da ferramenta computacional para simular um biofiltro de leito fluidizado, subsidiando o entendimento das variáveis importantes neste escoamento. A modelagem foi realizada utilizando-se a abordagem Euleriana-Euleriana e a Teoria Cinética de Escoamento Granular. Os resultados obtidos foram expressos através da análise de pressão e da fração volumétrica nos diferentes tempos analisados.

\section{INTRODUÇÃO}

A aquicultura tem aumentado intensamente nos últimos anos, em âmbito global, a produção deve crescer até alcançar 195,9 milhões de toneladas em 2025, um aumento de $17 \%$ em comparação a produção de 2013-15. Isso significa que no ano 2025 o mundo vai produzir 29 milhões de toneladas a mais de peixe que em 2013-15 e quase todo esse aumento vai acontecer nos países em desenvolvimento por meio da aquicultura (FAO, 2016). Para alcançar o desenvolvimento que se espera muitos desafios precisam ser superados pelo setor, a exemplo da utilização da água na aquicultura, alvo de preocupação mundial, pois as práticas de cultivo convencionais consomem grande quantidade de água de boa qualidade. Esse fato levou à busca por práticas alternativas para o cultivo de peixes e de tecnologias que levem à redução do consumo de água.

Comparativamente aos sistemas tradicionais de cultivo dos peixes em viveiros, os sistemas de recirculação proporcionam menor consumo de água por quilo de peixe produzido (redução de mais de 90\%) e como vantagem, apresentam baixíssima emissão de efluentes, reduzindo os impactos ambientais. A ampliação deste sistema de cultivo atende aos conceitos de uma aquicultura sustentável e ambientalmente correta (Blancheton, 2000; Azevedo et al., 2014). O uso dos biofiltros é fundamental para o tratamento das águas residuais na recirculação de sistemas de aquicultura (Recirculating Aquaculture Systems - RAS) porque a seleção de biofiltros influencia os custos de capital, de operação dos sistemas e a consistência do tratamento eficaz de água (Summerfelt, 2006). A nitrificação ocorre dentro de filtros biológicos quando dois grupos distintos de bactérias autotróficas catabolizam amônia não ionizada para nitrito e mineralizam nitrito em nitrato (Hagopian e Riley, 1998; Chen et al., 2006). Existem muitos tipos de biofiltros que são comumente usados em RAS, incluindo 


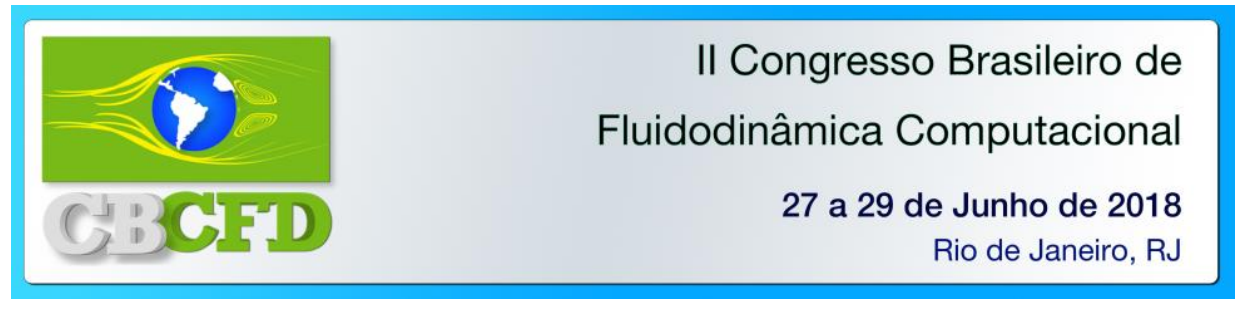

biofiltros submersos, biofiltros gotejantes, biofiltros de pérolas flutuantes, biofiltros de grânulos dinâmicos e biofiltros de leitos fluidizados (Timmons et al., 2002). Os biofiltros de areia fluidizada (Fluidized Sand Biofilters - FSBs) possuem tecnologia eficiente e competitiva em termos de custos para a remoção de resíduos dissolvidos nesses tipos de sistemas (Nam et al., 2000; Weaver, 2006). Como a mídia em biofiltros de areia fluidizada é de baixo custo e sua área superficial específica é maior, existem muitas vantagens, incluindo a alta eficiência no tratamento de água (remoção de amônia-azoto (TAN) de 50-90\%), tamanho pequeno e nenhuma necessidade de lavagem a contracorrente (Liu et al, 2015). O objetivo deste trabalho é utilização da ferramenta computacional para simular e otimizar um biofiltro de leito fluidizado, propondo variáveis adequadas ao efetivo controle e melhoria de eficiência desses equipamentos.

\section{METODOLOGIA}

O software utilizado foi o ANSYS FLUENT 18.2 e ANSYS CFX 18.2 (licença acadêmica). Sendo que ANSYS CFX 18.2 foi usado para a elaboração da geometria, malha e visualização dos resultados, e o ANSYS FLUENT 18.2 foi usado para a instalação das condições de contorno e parâmetros de simulação no setup. As simulações foram realizadas em um computador com configuração de processador ${ }^{\circledR}$ Intel core i5, HD de $1 \mathrm{~Tb} \mathrm{MHz}$ e memória RAM de $8 \mathrm{~Gb}$. A geometria é uma simplificação 2D do biofiltro de areia fluidizada utilizado nos estudos Liu et al. (2015). A modelagem foi realizada utilizando-se a abordagem Euleriana-Euleriana que interpreta as fases matematicamente como contínuas e as equações de Navier-Stokes foram aplicadas as fases, sendo o acoplamento entre elas realizado pela pressão e pelo coeficiente de troca de interface. Os princípios da conservação de massa e de momento foram aplicados às fases líquida e particulada. Foi utilizada a Teoria Cinética de Escoamento Granular (Taghipuor et al., 2005; Armstrong \& Luo, 2010; Sant'Anna et al. 2015; 2016; 2017).

O sistema de Equações obtido foi resolvido através de modelagem mecanicista utilizando-se o método Phase Coupled SIMPLE para solução do acoplamento pressãovelocidade. Foi utilizada a discretização espacial de segunda ordem para todas as equações, exceto para a fração volumétrica, sendo esta discretizada pelo método QUICK. Para a discretização temporal foi utilizada a discretização de segunda ordem implícita. O modelo de turbulência utilizado neste trabalho foi o k-E (Sant'Anna et al., 2015; 2016; 2017). O coeficiente de restituição partícula-partícula foi estabelecido como sendo igual a 0.9 (Pain et al., 2001; Taghipour et al., 2005; Cornelissen et al., 2007 e Adamczyk et al.,2013). O regime

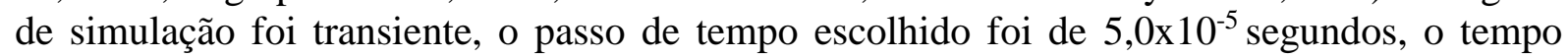
total de simulação foi de $5 \mathrm{~s}$, sendo necessárias 100.000 iterações para o teste de malha. Após

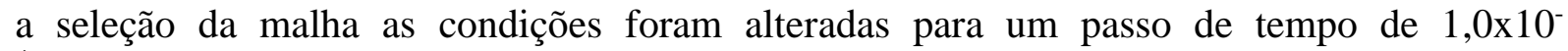
${ }^{4}$ segundos, $10 \mathrm{~s}$ de simulação. As propriedades da fase líquida (água) foram densidade e viscosidade com valores de $9,98 \times 10^{2} \mathrm{~kg} / \mathrm{m}^{3}$ e $10,03 \times 10^{-4} \mathrm{~kg} / \mathrm{ms}$ (ANSYS FLUENT, 2014), respectivamente. Para a fase particulada (areia) foram adotados os valores de $2,6 \times 10^{3} \mathrm{~kg} / \mathrm{m}^{3} \mathrm{e}$ $2,75 \times 10^{2} \mu \mathrm{m}$ para a densidade e o diâmetro da partícula (SANT'ANNA et al., 2017). A velocidade superficial da fase líquida durante a realização do teste de malha foi baseada em Liu et al. (2015), com o valor de $0,0271 \mathrm{~cm} / \mathrm{s}$.

Para o biofiltro o teste de malha foi realizado utilizando três níveis de refinamento crescente. A malha utilizada foi composta por elementos hexaédricos. Os dados estatísticos 


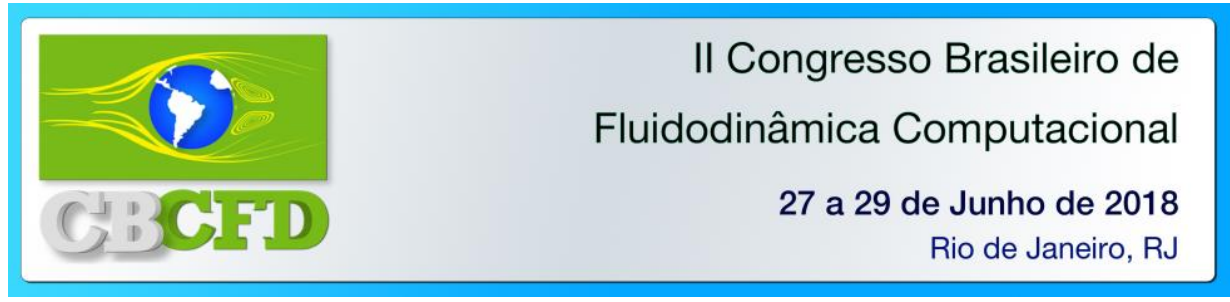

das malhas refinadas são apresentados na Tabela 1. Após a realização do teste de malha, foi necessário avaliar o comportamento do biolfiltro aumentando se a velocidade para $0,271 \mathrm{~m} / \mathrm{s}$.

Tabela 1- Estatística das malhas da geometria B.

\begin{tabular}{lccc}
\hline Malhas & Malha 1 & Malha 2 & Malha 3 \\
Parâmetros & & & \\
\hline Max face size & 0,005 & 0,003 & 0,002 \\
Número de nós & 21.076 & 58.482 & 130.750 \\
Número de elementos & 10.228 & 28.724 & 64.600 \\
Hexaedros & 10.228 & 28.724 & 64.600 \\
\hline
\end{tabular}

\section{RESULTADOS}

Para observar a influência da malha sobre os resultados foram escolhidas as variáveis fração volumétrica e queda de pressão. Foram construídas curvas de fração volumétrica de areia em função da altura do leito (Figura 1) e curvas para a queda de pressão ao longo da altura do leito para as diferentes malhas analisadas (Figura 2).

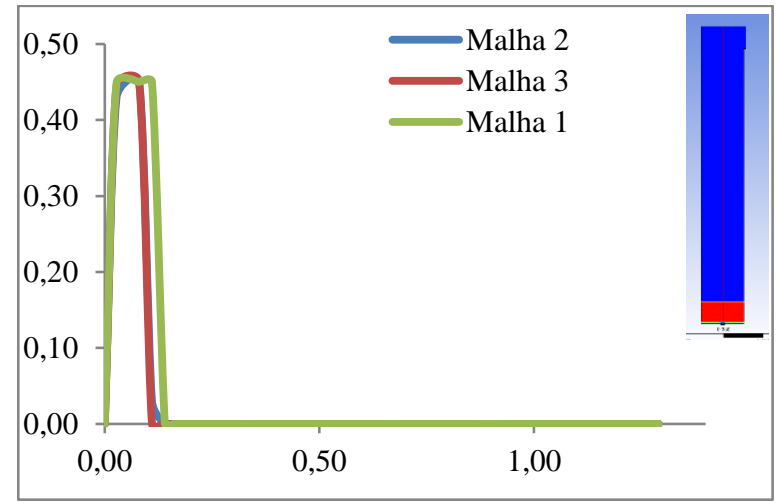

Figura 1 - Expansão do leito para diferentes malhas $(\mathrm{t}=2,50 \mathrm{~s})$.

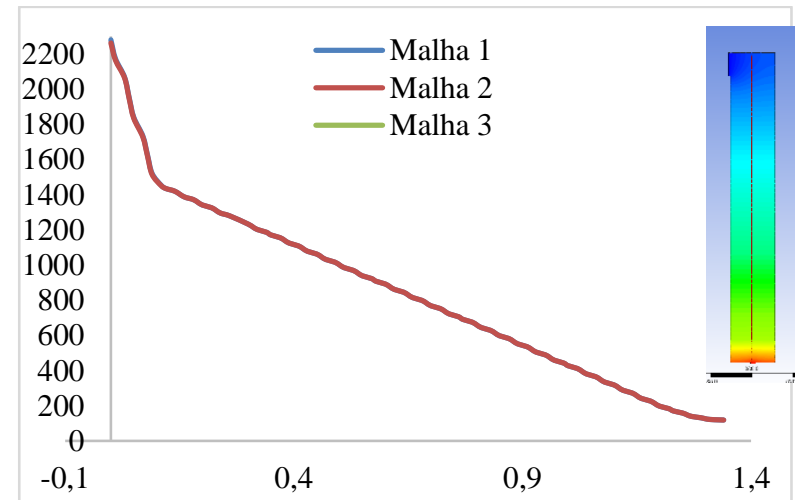

Figura 2- Queda de pressão versus altura do leito para diferentes malhas $(\mathrm{t}=2,50 \mathrm{~s})$.

Pode-se observar nas Figuras 1 e 2 que para as diferentes malhas testadas as curvas das Malhas 2 e 3 foram bem próximas, quase coincidentes para a fração volumétrica e superpostas para a queda de pressão. Optou-se, então, pela Malha 2, em virtude de esta apresentar um menor número de elementos e por consequência reduzir o esforço computacional. Para obter mais informações sobre esse escoamento, a malha 2 selecionada no teste de malha foi simulada para um tempo de $10 \mathrm{~s}$ com uma velocidade de $0,271 \mathrm{~m} / \mathrm{s}$ (dez vezes maior que a velocidade utilizada no teste de malha). Os perfis obtidos foram ilustrados na Figura 5 e a expansão do leito através dos diferentes tempos de simulação foi ilustrada na Figura 6. Ao analisar os perfis da Figura 5 e as curvas da Figura 6, foi possível verificar que a água promove o arraste da fase particulada ao longo do tempo, o leito tem altura inicial de $0,10 \mathrm{~m}$, alcança $0,56 \mathrm{~cm}$ (após os $5 \mathrm{~s}$ de simulação), no entanto essa expansão não é bem distribuída em toda a fase particulada, gerando picos de fração volumétrica de areia. O intuito do biofiltro é promover o contato bem distribuído entre as fases, de forma que o regime passe do leito fixo para o leito expandido. 


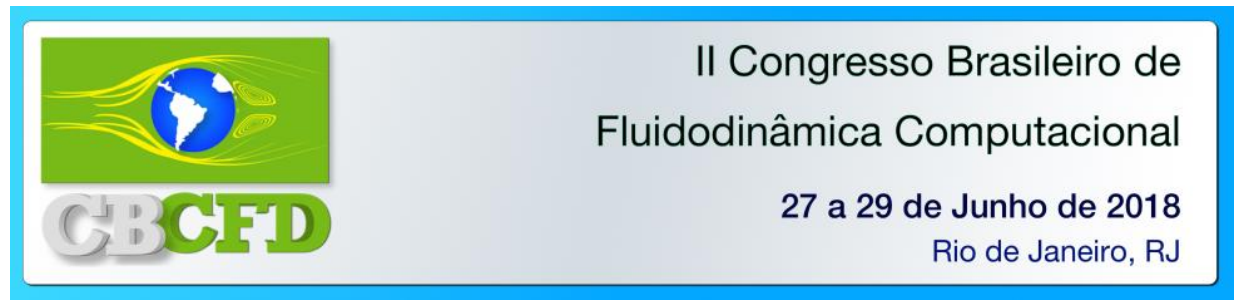

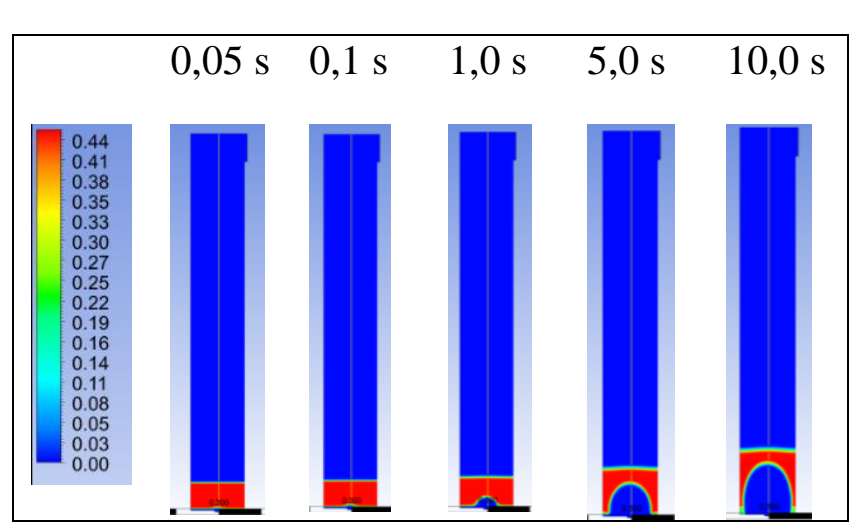

Figura 5 - Perfis longitudinais de fração volumétrica ao longo do tempo $\left(\mathrm{v}_{\mathrm{L}}=0,271 \mathrm{~m} / \mathrm{s}\right)$

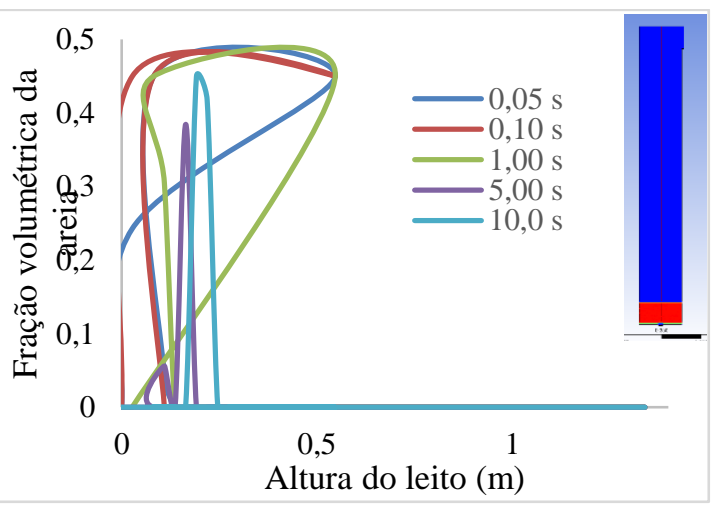

Figura 6 - Fração volumétrica $\left(v_{L}=0,271\right.$ $\mathrm{m} / \mathrm{s})$

Portando é necessário a realização de um planejamento fatorial, variando parâmetros importantes para a obtenção de uma condição de distribuição ótima da fase particulada através da fase liquida, promovendo assim um maior contato entre as fases e consequentemente maior superfície de contato, para a fixação das bactérias e promoção das reações de nitrificação.

\section{REFERÊNCIAS}

ANSYS, Theory Guide, Ansys Inc., USA, 2011.

ARMSTRONG, L.M.; GU, S.; LUO, K.H. Study of wall-to-bed heat transfer in a bubbling fluidised bed using the kinetic theory of granular flow. Int. J. Heat Mass Transf., vol. 53 p. 4949-4959, 2010.

AZEVEDO V. G.; NETO H. G.; ALMEIDA H. L. P. S.; SANCHES E. G. Sistemas de recirculação para peixes marinhos - Procedimento Operacional Padrão (POP), 2014.

BLANCHETON, J. P. Developments in recirculation systems for Mediterranea fish species. Aquacultural Engineering, vol 22 (1), 17-31, 2000.

CAVALCANTI, R. S. Estudo fluidodinâmico do desprendimento de $\mathrm{H}_{2}$ em uma célula eletrolítica utilizando o aplicativo computacional CFX, Dissertação de Mestrado, Universidade Federal de Campina Grande, Campina Grande/PB, Brasil, 2003.

CAVALCANTI, R. S.; FARIASNETO, S. R.; VILAR, R. E. A computational fluid dynamics study of hydrogen bubbles in an electrochemical reactor. Brazilian Archives of Biology Technology, v. 48, p. 219-229, 2005.

CORNELISSEN, J.T.; TAGHIPOUR, F.; ESCUDIÉA, R.; ELLIS, N.; GRACE, J.R. CFD modelling of a liquid-solid fluidized bed. Chem. Eng. Sci., vol. 62, p. 6334-6348, 2007.

FAO, El estado mundial de la pesca y la aquicultura 2008. Departamento de Pesca y acuicultura de la FAO. ROMA. p.197. 2009.

FAO, El estado mundial de la pesca y la aquicultura 2015. Departamento de Pesca y acuicultura de la FAO. ROMA, 2016. 